\title{
Meson photoproduction from the nucleon at CLAS
}

D.P. Watts for the CLAS collaboration at Jefferson Lab

SUPA, University of Edinburgh, UK

\begin{abstract}
The excitation spectrum of the nucleon provides a stringent constraint on the dynamics and interactions of its internal constituents and therefore probes the mechanism of confinement in the light quark sector. Our detailed knowlege of this excitation spectrum is poor, with many predicted states not yet observed in experiment and many "established" states having poorly known properties. To address these shortcomings a worldwide effort is currently underway exploiting the latest generation of electron and photon beams in detailed studies of meson photoproduction from nucleon targets. A major contribution to this effort will come from the experimental programme at Jefferson Lab exploiting the frozen spin target (FROST) with the CLAS spectrometer. The status of this project will be presented along with preliminary results and analyses.
\end{abstract}

\section{Introduction}

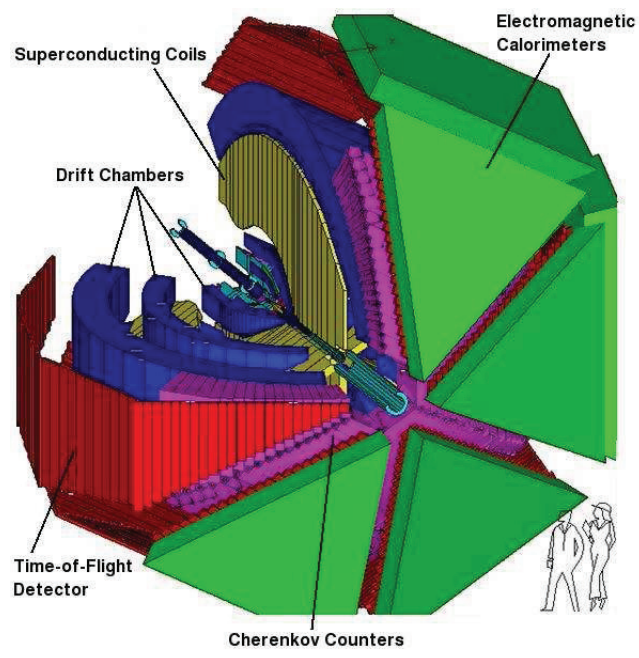

Fig. 1: Schematic diagram of the CLAS detector.

Obtaining an accurate determination of the excitation spectrum of the nucleon would provide stringent constraints on our understanding of the dynamics and degrees of freedom of its constituents. Significant advances in theory aiming to understand this spectrum have taken place in recent years. One recent highlight is the emergence and expected progression of Lattice QCD predictions, which provide a more direct link between the properties of the excitation spectrum and the underlying non perturbative QCD processes in the light quark sector [2]. Better establishing the excitation spectrum would also guide refinements to QCD-based phenomenological models such as constituent quark models. The recent lattice results tend to support the finding of many constituent quark models that there should be many more excited states of the nucleon than have currently been observed, particularly in the mass region above $1.8 \mathrm{GeV} / \mathrm{c}^{2}$. 


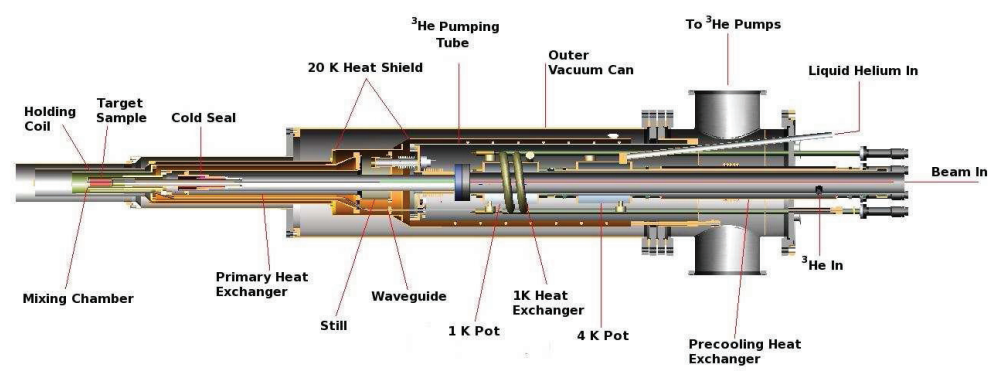

Fig. 2: Schematic diagram of FROST.

It remains a major challenge to current hadron physics to establish whether this "missing resonance" problem is due to deficiencies in the coverage, quality, or completeness of the world data set to date or whether these states are absent in nature, with significant consequences to our understanding of nucleon dynamics. As well as hunting for unseen resonances, it is important to better establish the properties of "known" resonances, reaching a situation where they are consistently evident in different analyses and have consistent electromagnetic couplings, masses and widths.

Meson photoproduction is an excellent tool to study the nucleon resonance spectrum. The photon offers a clean well understood probe, with the advantage over previous studies with spinless pion beams that the response of the nucleon to polarisation degrees of freedom can be utilised. The new generation of measurements of meson photoproduction carried out or planned at many of the world's electromagnetic beam facilities will provide a step change in the quality of available experimental data. The use of photon beams and targets with high degrees of polarisation, coupled with large acceptance particle detectors is essential for disentangling the spectrum of excited states, which can only be revealed from the data by a detailed partial wave analysis (PWA). The inadequacies of the current world data set are highlighted by the differences in the composition of the resonance spectrum by different partial wave analyses of the same data (for a review see [3]). Significant advances in PWA analyses themselves are also currently underway, including the SAID [5], MAID [6], Bonn-Gatchina [7] and EBAC [1]. One recent highlight from these developments comes from the coupled channel models developed by the EBAC group at Jefferson Lab, which recently led to the suggestion that the low mass of the Roper resonance may arise from strong coupled channel effects [1]. A common problem for all the PWA's in meson photoproduction is that they are currently under-constrained and require more comprehensive experimental data to permit more consistent interpretations of the contributing resonances and reaction mechanisms.

The CLAS measurements presented in this contribution will form a crucial part of this world effort to improve the world data base available for PWA studies. The FROST experiment will provide complete or nearly complete measurements for a whole range of meson photoproduction reactions such as $\gamma p \rightarrow N \pi, p \eta, p \omega, K^{+} Y$ and $p \pi^{+} \pi^{-}$. A "complete" measurement corresponds to the determination of sufficient experimental quantities to eliminate experimental ambiguities in extracting the scattering amplitude for the reaction and thereby putting as tight constraints on the various PWA's as possible. Achieving a complete measurement requires the precise determination of at least eight well chosen experimental observables (for a recent discussion see [4]). One combination is the cross section, three 
single-spin and four double-spin observables. For hyperon production channels the ability to determine the polarisation of the recoiling hyperon from the angular distribution of its weak decay allows this complete determination to be achieved entirely from observables measured in the CLAS programme. For non-strange production channels a close to complete set can be obtained at CLAS.

The experimental facilities will be discussed in section 2. A selection of preliminary results for beam-target observales will be presented in sections 3-6.

\section{Experimental Setup}

The Continuous Electron Beam Accelerator Facility (CEBAF) provides high-intensity electron beams of up to $6 \mathrm{GeV}$ with approximately $100 \%$ duty cycle simultaneously to three experimental halls: A, B and C. The upgrade to $12 \mathrm{GeV}$ will be completed in 2014, with associated upgrades to the equipment in the three current experimental halls and a fourth hall, D, currently under construction. The CEBAF accelerator consists of two anti-parallel superconducting RF-linacs connected by recirculation arcs to create a racetrack with a combined length of $1.4 \mathrm{~km}$. The accelerator produces a continuous-wave electron beam which can circulate around the linacs up to 5 times, gaining $1.2 \mathrm{GeV}$ of energy with each pass.

Hall B houses the CEBAF Large Acceptance Spectrometer (CLAS), which can be used with both electron and photon beams. A schematic diagram of CLAS is shown in Fig.1. CLAS is a multi-layered magnetic spectrometer, divided into six independent sectors by six superconducting coils arranged symmetrically around the beamline producing a toroidal magnetic field. There is a magnetic field free region in the centre of the detector, allowing for the use of polarised targets such as FROST. For photon beam experiments the innermost detector is a scintillator start counter surrounding the target providing timing and triggering information on charged reaction products. Outside the start counter, the first three layers of detectors consist of drift chambers to determine the curved trajectories and hence momenta of charged particles. Surrounding the drift chambers, gas Cherenkov Counters identify electron events and allows for the differentiation of electrons from pions. This is then surrounded by a layer of plastic scintillation bars for time-of-flight measurements. The outermost detector system is made up of electromagnetic calorimeters for the detection of photons, electrons and high-energy neutrons.

Near the entrance to Hall B the electrons from CEBAF impinge on a thin radiator in which they undergo bremsstrahlung. The recoiling electrons from this process are momentum analysed in a magnetic spectrometer containing a highly segmented focal plane comprised of thin plastic scintillation detector elements. The device can tag photons in the energy range 0.35 to $5.8 \mathrm{GeV}$. Circularly polarised photons can be produced by utilising a longitudinally polarised electron beam. The direction of the electron polarisation (and therefore photon circular polarisation) was flipped at a rate of $30 \mathrm{~Hz}$. Linear photon polarisation can be achieved by using a thin diamond radiator. For such cases the lattice structure coherently enhances the momentum transfer direction in the scattering process to directions related to the reciprocal lattice vectors of the crystal, producing linear polarisation for the bremsstrahl photons.

The frozen spin target (FROST) is placed at the centre of the CLAS detector and provides a highly polarised proton target for use in a wide range of experiments. A schematic of the FROST apparatus is shown in Fig. 2. The target material consists of frozen, 1-2 mm beads of butanol $\left(\mathrm{C}_{4} \mathrm{H}_{9} \mathrm{OH}\right)$ mixed by weight with $5 \% \mathrm{H}_{2} \mathrm{O}$ and $0.5 \%$ TEMPO (2,2,6,6-tetramethylpipendine- 1-oxyl), and is placed inside the target cup which is $50 \mathrm{~mm}$ long and $15 \mathrm{~mm}$ in diameter. The target material was chosen to have a high maximum polarisation, a high ratio of polarisable nucleons to the total number of nucleons (the quality factor), a high resistance to ionising radiation, and a small number of unwanted polarisable nuclei. In butanol the non-hydrogen components, carbon and oxygen, are spinless particles, so pure butanol has no background polarisation to correct for. To subtract the yield from the unpolarised nuclei the frost target was run simultaneously with a separate carbon and polythene detector downstream of FROST. Events from these targets could be identified from the vertex tracking information of CLAS and their contribution to the butanol yield could be assessed for any variable of interest in the analysis. In operation 
the FROST target achieved polarisation levels immediately following the polarising process of $85 \%$ to $90 \%$ and the polarisation degraded at a rate of $0.9 \%$ (spin paralell to beam) to $1.5 \%$ (anti-parallel to beam axis) per day.

In the following sections some of the preliminary analysis results from the first round of FROST data using a longitudinally polarised proton target will be presented.
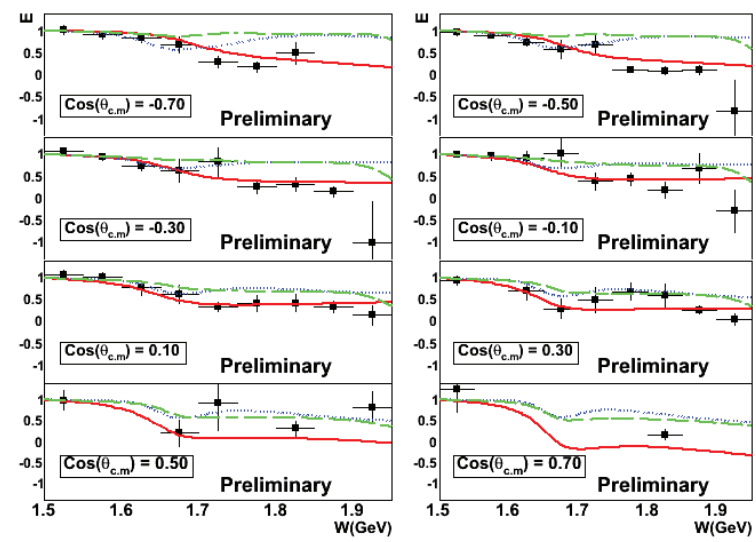

Fig. 3: Preliminary results for $E$ in $\vec{\gamma} \vec{p} \rightarrow p \eta$ for energies $W=1.525-1.925 \mathrm{GeV}$. Curves: $\eta$-MAID (dotted line), Bonn-Gatchina PWA (dashed line), and SAID (solid line).
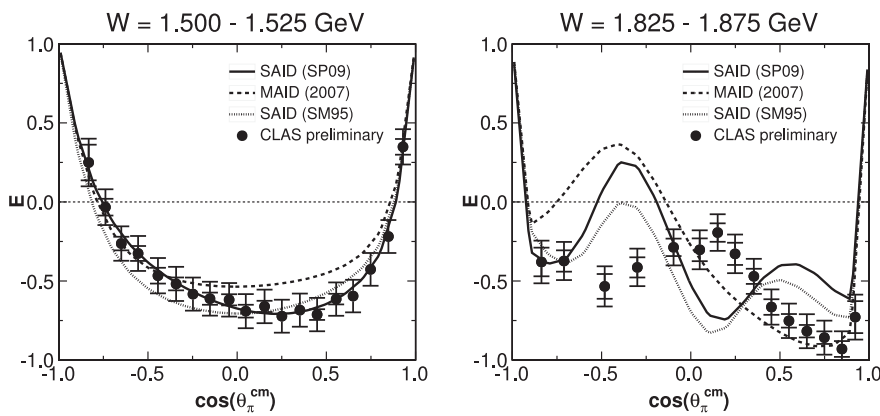

Fig. 4: Preliminary results of the double-polarisation observable $E$ (helicity difference) for $\vec{\gamma} \vec{p} \rightarrow n \pi^{+}[11]$. The inner error bars indicate stat. uncertainties; the outer error bars include a $10 \%$ sys. uncertainty, which is expected to be reduced in the final analysis. The curves show solutions of the SAID SP09 [5], MAID [6] and SAID SM95 PWA.

\section{The beam-target helicty asymmetry, E, in $\eta$ photoproduction}

The measurement of a wide range of final states in meson photoproduction is highly desirable, not only to constrain coupled channel analyses but also as certain channels are expected to filter contributions of nucleon resonances to the scattering amplitude. One example of this is $\eta$ photoproduction which due to the $\mathrm{I}=0$ isospin of the $\eta$ can be produced in a single step mechanism only via intermediate nucleon resonances having isospin, $\mathrm{I}=\frac{1}{2}$. This restriction simplifies the data interpretation and theoretical efforts to predict the excited states contributing to these reactions. 

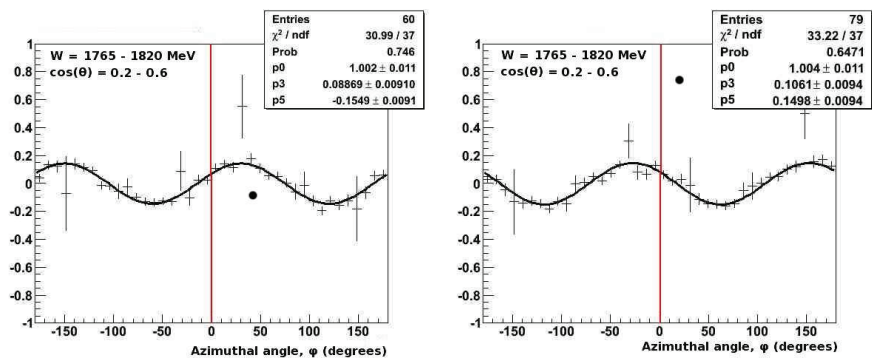

Fig. 5: (Upper) phi asymmetry for the positively polarised target setting. (Lower) phi asymmetry for negative target setting. The vertical red line at $0^{0}$ is present to help demonstrate the phase shift in the asymmetry due to the $\mathrm{G}$ contribution to the cross section [8]
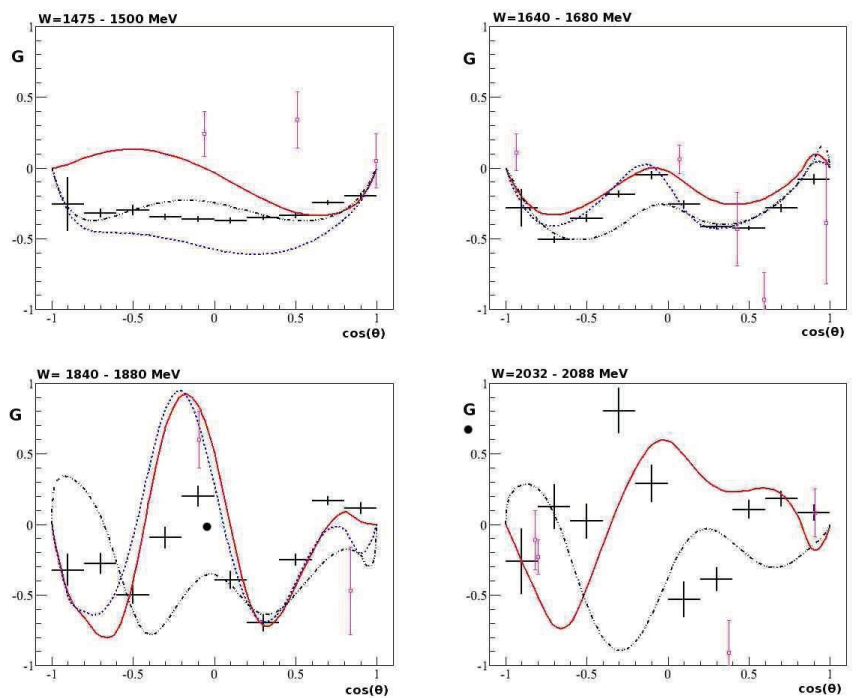

Fig. 6: Preliminary FROST values of the double-polarisation observable $G$ (black points) plot as a function of $\cos (\theta)$ for a small fraction of the available mass (W) bins [8]. Overlaid on the plots are the current SAID [5] (solidred line) and MAID2007 [6] (dashed-blue line) solutions. The open pink squares show the previous experimental data for $G$.

The polarised cross section for the reaction $\vec{\gamma} \vec{p} \rightarrow p \eta$ of circularly-polarised photons on longitudinallypolarised protons is given by:

$$
\frac{d \sigma}{d \Omega}=\frac{d \sigma}{d \Omega}\left(1-P_{z} \delta_{\odot} E\right)
$$

where $d \sigma / d \Omega_{0}$ is the unpolarised cross section. $P_{z}$ and $\delta_{\odot}$ are the degrees of target and beam polarisation, respectively. $E$ denotes the helicity asymmetry.

The asymmetry is extracted from the difference in reaction yields between the photon and target nucleons spins aligned or antialigned. Preliminary results [9] of the helicity asymmetry, E, for $\vec{\gamma} \vec{p} \rightarrow p \eta$ are shown in Fig. 3. Since the $\eta$ threshold is dominated by the $\mathrm{N}(1535) \mathrm{S}_{11}$ resonance, the observable exhibits values close to unity for $\mathrm{W} \leq 1.6 \mathrm{GeV} / \mathrm{c}^{2}$. The preliminary results indicate that the observ- 


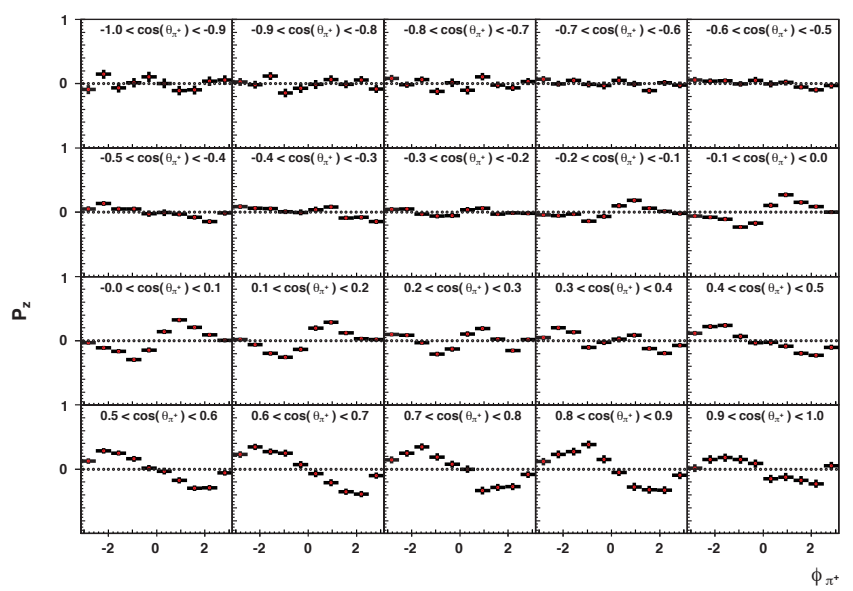

Fig. 7: Preliminary target asymmetry, $P_{z}$, from FROST for the reaction $\vec{\gamma} \vec{p} \rightarrow p \pi^{+} \pi^{-}$for $E_{\gamma} \in$ $[0.7,0.8] \mathrm{GeV}[10]$. Error bars are statistical only.

able remains positive below about $\mathrm{W}=2 \mathrm{GeV}$, providing new information to constrain the partial wave analyses for this channel.

\section{The beam-target helicity asymmetry $\mathrm{E}$ in $\pi^{+}$photoproduction}

Comprehensive measurement of the pion production reaction is important as, of all the production channels accesible, the pion photoproduction is expected to give sensitivity to the largest set of resonances. The current database for pion photoproduction is mainly populated by unpolarised cross section data and single-spin observables and is therefore significantly under-constrained.

Figure 4 shows preliminary results [11] of the double polarisation E for $\vec{\gamma} \vec{p} \rightarrow \pi^{+} n$ (Eqn. 1). While the predictions shown in Fig. 4 agree nicely with the new data at low energies, discrepancies emerge at higher energies for $\mathrm{W} \geq 1.7 \mathrm{GeV} / \mathrm{c}^{2}$. Single-pion photoproduction in this region appears less well understood than previously expected. The implications for the resonance spectrum and properties will be explored when the finalised results are included in the various PWAs.

\section{The beam-target linear asymmetry $G$ in $\pi^{+}$photoproduction}

The use of a linearly polarised photon beam with a longitudinally polarised proton target gives access to the observable $\mathrm{G}$, for which only sparse data exists in the current world data base. The differential cross section for the case of a linearly polarised photon beam and longitudinally polarised proton target can be written as:

$$
\frac{d \sigma}{d \Omega}=\left(\frac{d \sigma}{d \Omega}\right)_{0}\left(1-P_{L} \Sigma \cos (2 \phi)+P_{L} \delta_{\odot} G \sin (2 \phi)\right),
$$

where $d \sigma / d \Omega_{0}$ is the unpolarised cross section. $P_{L}$ and $\delta_{\odot}$ are the degrees of linear beam and target polarisation, respectively. $\mathrm{G}$ denotes the beam-target linear asymmetry observable and $\Sigma$ is the single beam polarisation observable.

There were two possible settings for the photon beam polarisation in the first FROST experiment: PARA $(\|)$ in which the electric field vector was parallel to the floor and PERP $(\perp)$ in which the electric 
field vector was perpendicular to the floor. In addition, the target was polarised either parallel or antiparallel to the beam direction, defined as positive and negative target polarisation, respectively. The combination of these resulted in a total of four possible combinations of polarised beam-polarised target settings during the run. An asymmetry in the reaction yield as a function of pion azimuthal angle with respect to the photon polarisation plane can be obtained:

$$
\frac{N(\phi)_{\perp}-N(\phi)_{\|}}{N(\phi)_{\perp}+N(\phi)_{\|}}=P_{L} \Sigma \cos (2 \phi)-P_{L} \delta_{\odot} G \sin (2 \phi) .
$$

The value of $\mathrm{G}$ could be extracted by fitting these phi asymmetry spectra. Typical plots for positive and negative target polarisation settings are shown in Fig. 5. The effect of $G$ in equation (3) gives the expectation of a change in sign of the $\phi$ offset when the target is polarised in the positive or negative direction. This is clearly seen when comparing the 2 plots in the Fig. 5 .

Preliminary values for $\mathrm{G}$ for a sample of the available $\mathrm{W}$ bins are shown in Fig. 6. It should be noted that the systematics in the degree of linear polarisation of the beam are still being evaluated so the results are at an early stage. Despite the preliminary nature it is clear that the new data show a great improvement in accuracy and kinematic coverage compared to previous measurements. Even in the low lying part of the excitation spectrum near to the Roper resonance it is seen that current PWAs give different expectations for $\mathrm{G}$. These significant differences highlight how the current world data does not constrain this reaction even in the low lying part of the spectrum. The new data will therefore give valuable new constraints on PWAs over the mass range from 1400 up to $2300 \mathrm{MeV}$.

\section{Polarisation observables for $\pi^{+} \pi^{-}$production on the proton}

One of the key experiments in the search for yet unobserved states is the investigation of double-pion photoproduction. Quark models predict large couplings of those states to $\Delta \pi$, for instance. The fivedimensional cross section for the photoproduction of two pseudoscalar mesons using longitudinal target polarisation and circularly-polarised (or unpolarised) beam can be written in the form [12]:

$$
I=I_{0}\left\{\left(1+\Lambda_{z} \cdot P_{z}\right)+\delta_{\odot}\left(I^{\odot}+\Lambda_{z} \cdot P_{z}^{\odot}\right)\right\}
$$

where $I_{0}$ denotes the unpolarised cross section and $\delta_{\odot}$ and $\Lambda_{z}$ denote the degree of beam and target polarisation, respectively. The additional polarisation observables, $P_{z}$ and $I^{\odot}$, for the two-meson final state arise since the reaction is no longer restricted to a single plane.

Figure 7 shows an example for the observable $P_{z}$ in $\gamma^{\rightarrow} p \rightarrow \pi^{+} \pi^{-}$[10]. The variables $\theta$ and $\phi$ denote the polar and azimuthal angle of the $\pi^{+}$in the rest frame of the two mesons. The observable acquires surprisingly large values for positive $\cos \theta_{\pi^{+}}$with the statistical errors in some cases smaller than the symbol size. The expected odd behaviour of the distribution is clearly visible.

\section{Conclusion}

Nucleon spectroscopy is undergoing an exciting period with major advances in theory being complemented by a step change in the quality of experimental data. Experimental programmes of meson photoproduction measurements at the world's electromagnetic beam facilities will provide the long sought extensive data sets to constrain the various partial wave analyses and more reliably extract the excitation spectrum of the nucleon and its properties. The FROST programme at Jefferson Lab will provide a large proportion of this new high quality experimental data. In the coming decade the combined efforts of experimentalists and theorists in this area is expected to allow deeper insights into our understanding of QCD confinement in the light quark sector. 


\section{Acknowlegements}

The work is supported by DOE and NSF grants. The author thanks the Science and Technology Funding Council (UK) for support.

\section{References}

[1] H.Kamano and S. X. Nakamura and T. -S. H. Lee T. Sato, Phys. Rev. C81, 065207 (2010).

[2] Bulava, J. and Edwards, R. G. and Engelson, E. and Joó, B. and Lin, H-W. and Morningstar, C. and Richards, D. G. and Wallace, S. J Phys. Rev. D82, 014507 (2010); arXiv:1104.5152 [hep-ph]

[3] E. Klempt, J-M Richard, Rev. Mod. Phys. 82, 1095 (2010)

[4] B. Dey, M.E. McCracken, D.G. Ireland, C.A. Meyer, Phys Rev C83 055208 (2011)

[5] M. Dugger et al. [CLAS Collaboration], Phys. Rev. C79, 065206 (2009) and references therein; http://gwdac.phys.gwu.edu/

[6] D. Drechsel, O. Hanstein, S. S. Kamalov, L. Tiator, Nucl. Phys. A645, 145-174 (1999); http://wwwkph.kph.uni-mainz.de/MAID/

[7] E. Klempt, A.V. Anisovich, V.A. Nikonov, A.V. Sarantsev, U. Thoma., Eur.Phys.J.A24:111128,2005; http://pwa.hiskp.uni-bonn.de/

[8] J. McAndrew, Ph.D thesis University of Edinburgh, UK, 2011 submitted

[9] B. Morrison, NSTAR 2011, Jefferson Lab.

[10] S. Park, NSTAR 2011, Jefferson Lab;

[11] S. Strauch, arXiv:1108.3050 [nucl-ex].

[12] W. Roberts, T. Oed, Phys. Rev. C71, 055201 (2005). 